\title{
Interventions to prevent needle stick injuries among health care workers
}

\author{
Henk F. van der Molen ${ }^{1 \mathrm{a}}$, Koos AH Zwinderman ${ }^{\mathrm{b}}$, Judith K. Sluiter ${ }^{\mathrm{a}}$, Monique H.W. Frings-Dresen ${ }^{\mathrm{a}}$ \\ ${ }^{a}$ Coronel Institute of Occupational Health, Academic Medical Center, University of Amsterdam, PO Box 22700 , \\ 1100 DE Amsterdam, the Netherlands \\ ${ }^{b}$ Clinical Epidemiology and Biostatistics, Academic Medical Center, University of Amsterdam, P.O. Box 22700, \\ 1100 DE Amsterdam, The Netherlands
}

\begin{abstract}
Needle stick injuries (NSIs) are frequently reported as occupational injuries among health care workers. The health effects of a NSI can be significant when blood-to-blood contact occurs from patient to health care worker. The objective of this study was to evaluate whether the number of NSIs decreased among health care workers at risk in one Dutch academic hospital after introduction of injection needles with safety devices in combination with an interactive workshop. In a cluster threearmed randomized controlled trial, 23 hospital divisions ( $\mathrm{n}=796$ health care workers) were randomly assigned to a group that was subjected to the use of a 'safety device plus workshop', to a group that was subjected to a 'workshop only' or to a control group with no intervention. The combined intervention of the introduction of needle safety devices and an interactive workshop led to the highest reduction in the number of self-reported NSIs compared to a workshop alone or no intervention. For practice, the use of relatively simple protective needle safety devices and interactive communication are effective measures for reducing NSI's.
\end{abstract}

Keywords: needle stick injuries, prevention, health care

\section{Introduction}

Worldwide, needle stick injuries (NSIs) are frequently reported as occupational injuries among health care workers. Twelve-month NSI incidences up to $80 \%$ are reported among health care workers [e.g. 1]. The health effects of a NSI can be significant when blood-to-blood contact occurs from patient to health care worker.

The objective of this study was to evaluate whether the number of NSIs decreased among health care workers at risk in one Dutch academic hospital after introduction of injection needles with safety devices in combination with an interactive workshop or after an interactive workshop only compared with a control group with no intervention [2].

\section{Method}

In a cluster three-armed randomized controlled trial (RCT), 23 hospital divisions, were randomly assigned to an intervention group that was subjected to the use of a 'Needle safety device plus a Workshop' (NW group; seven wards, $\mathrm{n}=267$ ), to an intervention group that was subjected to a 'Workshop only' (W group; eight wards, $\mathrm{n}=263$ ) or to a 'Control group' with no intervention ( $\mathrm{C}$ group; eight wards, $\mathrm{n}=266$ ). Health care workers from the hospital wards were followed in the three groups for 12 months and their half-year incidences of NSIs were compared. Questionnaires and an official hospital notification registry were used as measurement instruments at three time points: baseline, 6 months follow-up and 12 months follow-up.

\footnotetext{
${ }^{1}$ Corresponding author. E-mail: h.f.vandermolen@amc.nl
} 


\subsection{Interventions}

The injection needle with a safety device (see Fig. 1), which was selected by the occupational health service of the academic hospital, was introduced through the supplier of this device. The supplier demonstrated the new working method for the injection needles with safety devices after the last workshop (see Fig. 2) and during the official breaks of that day at each ward. The day after the demonstration, all of the existing injection needles on the ward were replaced by the new injection needles with the safety device.

The workshop consisted of a one-hour interactive PowerPoint presentation where participants of one ward were informed and information was exchanged with each other about the prevalence, causes, consequences and prevention of NSIs in their hospital wards.

\subsection{Statistics}

The number of self-reported NSIs during the previous 6 months for each respondent was dichotomized (no versus $\geq 1$ ) for each time of measurement. Differences in the proportion of respondents with NSIs between the intervention groups and the control group for each time of measurement were examined using GEE correcting for wards. Changes in the proportion of workers with NSIs during follow-up (T1 and T2) were analyzed using GEE with wards and workers as cluster variables and the type of intervention, time and their interaction. The effects of both interventions were compared with the control group using odds ratios.

The number of official hospital register-based NSIs during the previous 6 months was converted into ward level. For calculating the NSI incidence, the number of included workers at baseline per ward was used as the denominator. Differences in the proportions of respondents with NSIs between the intervention groups and the control group for each time of measurement were examined using cross sectional non-parametric Kruskal-Wallis tests.

All tests were carried out using SPSS (Statistical Product and Service Solutions version 15.1). A significant difference was defined as $\mathrm{p}<0.05$.

\section{Results}

A statistically significant difference was found between the intervention groups and the control group for the half-year incidence of self-reported NSIs for

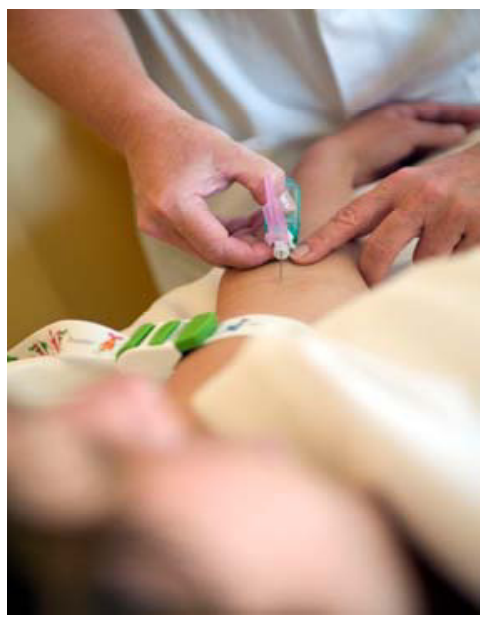

Figure 1. Injecting with a safety needle

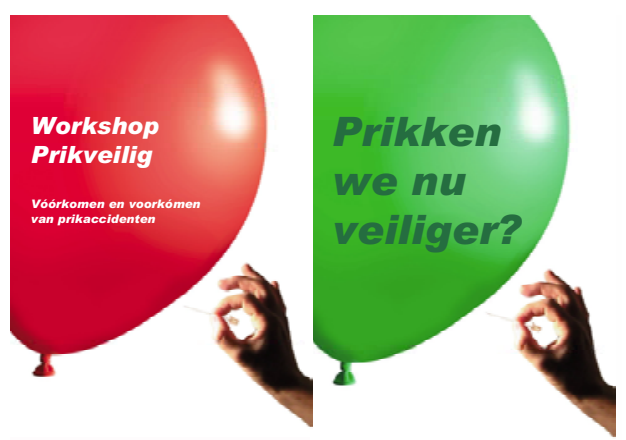

Figure 2. Workshop slide

the NW group (OR: 0.34 (95\% CI: $0.13-0.91))$ and for the W group (OR: 0.45 (95\% CI: $0.19-1.06)$ ) [2]. The officially registered NSIs during the study period showed no statistical differences between the groups.

\section{Discussion}

The combined intervention of the introduction of needle safety devices and an interactive workshop led to the highest reduction in the number of selfreported NSIs.

Method triangulation by means of the official hospital-based registration of NSIs was meant to reduce the risk of reporting bias. Notifications by official 
hospital-based registers, however, are also selfreported and likely to underestimate the actual occurrence of NSIs [3]. No reduction in the number NSIs as registered in the official hospital database could be due to a lack of compliance to systematically report to the occupational health service and the cross sectional analysis of these data.

The intervention measures (needle safety devices and workshops) and implementation strategies (facilitation and education) were developed by the occupational health service in dialogue with the management of the wards, purchasing and laboratory staff. This approach enabled the stakeholders, who also aimed to be involved in implementing the recommendations of the study, to discuss and consider the optimal intervention and implementation strategy to reduce NSI.

In conclusion, the use of relatively simple protective needle safety devices and interactive communication are effective measures for reducing NSI's. In these interactive communications, additional safety measures, such as good housekeeping, training and the use of personal protective equipment should be discussed and implemented.

\section{References}

[1] Smith DR, Choe MA, Jeong JS, Jeon MY, Chae YR, An GJ. Epidemiology of needlestick and sharps injuries among professional Korean nurses. J Prof Nurs (2006), 22, 359-366.

[2] van der Molen HF, Zwinderman KAH, Sluiter JK, FringsDresen MHW. Better effect of the use of a needle safety device in combination. Safety Science (2011), 49, 1180-1186.

[3] Schmid K, Schwager C, Drexler H. Needlestick injuries and other occupational exposures to body fluids amongst employees and medical students of a German university: incidence and follow-up. J Hosp Infect (2007), 65, 124-130. 